\title{
Biomecanical changes of the temporomandibular joint in restoration with implant supported overdentures. Functional analysis
}

\author{
Cristina Adriana Dahm Tataru ${ }^{1 *}$ \\ ${ }^{1}$ Lucian Blaga University, Department of Dental Medicine and Nursing Bdul Victoriei 10, Sibiu, \\ Romania
}

\begin{abstract}
Temporo-mandibular joint disorders represent an issue of particular importance for the health and proper functioning of the dentomaxillary systhem. Research conducted in this paper aims to analyse biomechanics and temporomandibular joint among patients who, one the one side have implants placed over a history of joint disease, emphasising developments along post-interventio. On the oder side, patients without a history of joint pathology were recieving implant prosthesis. The study includes a total of 138 subjects $(\mathrm{N}=138)$, age between 18 and 64 years, with lateral edentulous that have been applied to dental implants and made overdenture. All patients are clinically healthy. The research methods comprise systematic observation and clinical trial, applied to two groups: one of study and one of control. Biomechanical principles can not guarantee the avoidance in the case of biopathological response to overload (respectively outer-implant bone resorption). These will not lead to the prognostic for all types of prosthetic overstructures with implant support, because the individual variations of bone resistance can not be apropriatly measured. The present paper is an applied research which is designed to solve short term problems, in order to contribute to the development of an interdisciplinary scientific field.
\end{abstract}

\section{Introduction}

The biodynamics of the temporomandibular joint has been studied since ancient times, because the types of mastication have always imposed a series of changes with an extremely vast and painful pathology. In order to restaurate the masticatory function that was destroyed by the presence of the edentulous breaches for a longer or shorter period of time, requires a deep knowledge of the biomechanics of the temporomandibular joint. Also, modern systems of recording the individual characteristics of the case, (represented especially by articulators and facial arches), allows an efficient registration of parafunctions and / or masticatory dysfunctions and thus, the manufacture of correct and effective prosthetic works, which can restore the masticatory dysfunction in a fairly short time - in most situations.

\footnotetext{
*Corresponding author: info@dentalsibiu.ro
} 
Chronic degenerative changes of the temporo-mandibular joint including periarticular structures have a very high incidence among the population, the vast majority, still suffering from an early age in a lesser or greater extent of this condition (Figure 1). Most diseases are characterized by a worsening trend over many years, being more obvious with age.

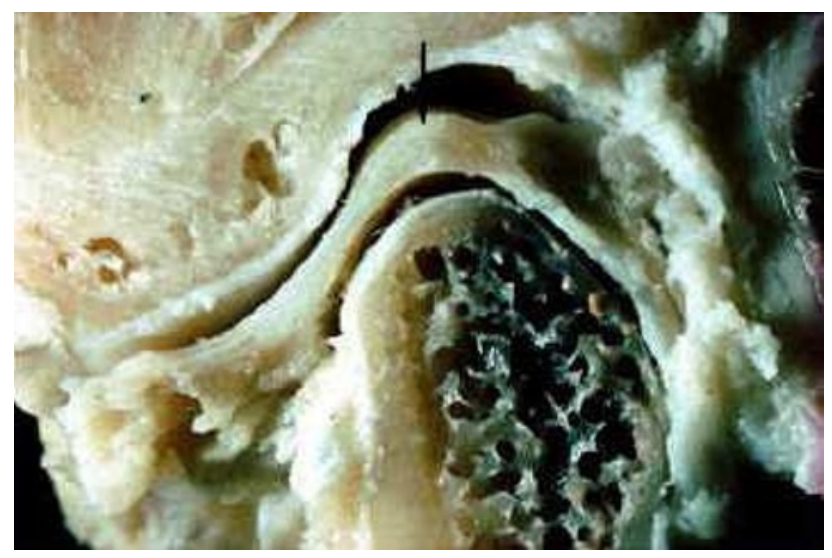

Fig.1. The temporomandibular joint.

However, following the biomechanical principles can not guarantee that the biopathological response to overload will be avoided. The main condition to make a biodinamic ideal superstructure is a static position in the dental arch with favorable spatial links. Specimens will be fixed in the articulator and will be marked with the ideal implant position and orientation. Also, will be a preoperative planned layout of the superstructure to allow considerations of biomechanical system and occlusion.

Bilateral edentulous leads to biomechanical changes at the temporomandibular joint level (and this is the main situation that we have to handel in our study). Those are the results of joint anatomy changes, such as the periarticular muscle, condyle and meniscus. [1]

Analysing the biomechanical principles applyed in clinical dentistry jaw movements, especially in terms of functional occlusal relationships can be followed by studying the biomechanics of the jaw at the level of 3D- movements and jaw positions.

Any jaw movement, is the result of mobilizing all groups that operate in couples as protagonists and antagonists.

This can be accomplished by grading the contraction force, by allowing the alternate contractions bilaterally symmetrical and asymmetrical unilateral and by the fact that these muscles may act on several fronts. [2-7]

Mandibular movements, in anatomic and functional terms can be simplified to three types of bidirectional movements, namely: lifting and lowering, propulsion and retropulsion, lateropulsion (right and left).

\section{Material and methods}

Data from the study of literature and clinical experience to date has led us to observe a large number of patients with masticatory disfunctions, of certain invoices, that we have taken into account in selecting the study group.

The research was conducted on a sample of 400 subjects age 18 to 60 years, of which 138 are in the experimental group.

The experimental group consists of pacients with lateral edentations witch received implant supported overdentures. The main problem of this group is the fact that in the period 
of time in witch they where edentulous -between 6 month and 2 years, we cand register specific changes at the temporomandibular joint level.

We intend to study a possible cause of disease determined by the type of mastication. There are two types of mastications: the bilateral alternativ mastication (this is the reccomended type of mastication because it leads to the minimum stress for the two temporomandibular joints) and the unilateral mastication witch occures in pathological situations ore bad habbits.

Most of the pacients that we examed in our study $(51,68 \%)$ used the bilateral alternativ mastication. It was unexpected, because the forming of the food bowl is done in the molar area but exactly this area is in this case not functional (Figure 2). That means that this action is replaced by the premolar area, but this is the topic of a future study.

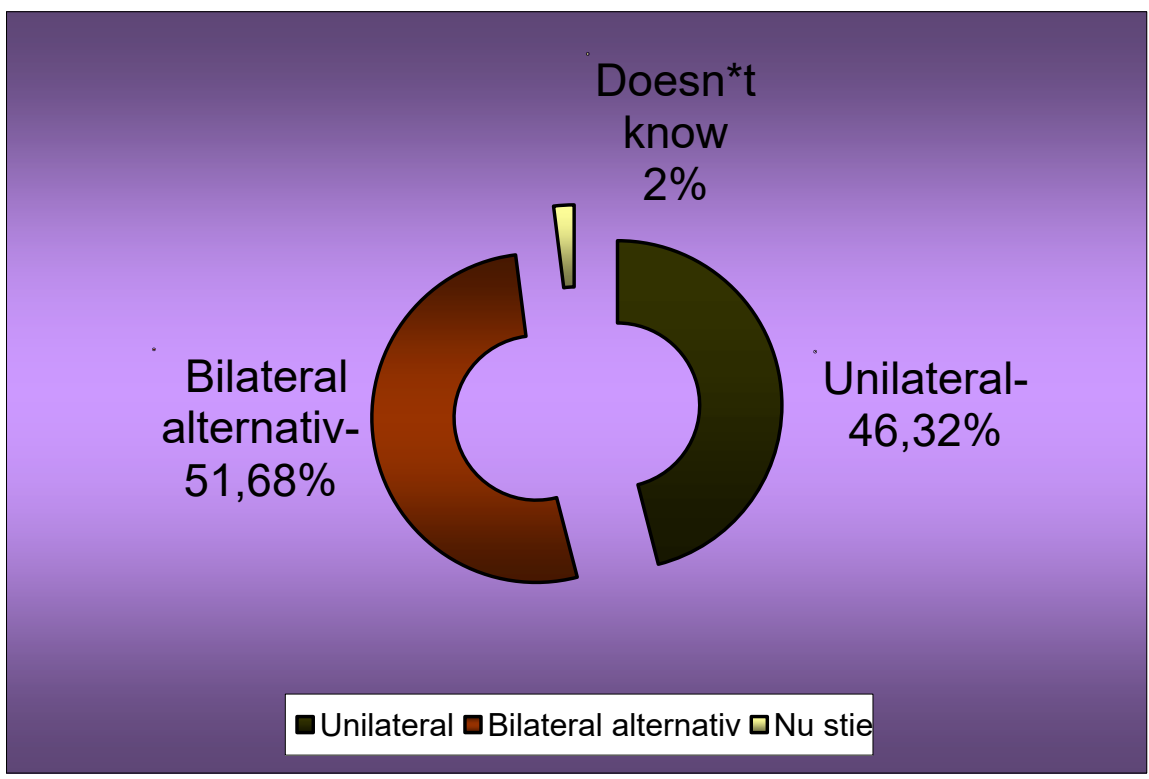

Fig. 2. Mastication type.

Once articular pathology occures we noticed very often -at the first level of the pathology, a dislocation of the articular meniscus, during the masticatory movements.

In the second level of the articular pathology, we can registrate a number of changes in the joint anatomy. This will lead to changes of the masticatory movements.

It is mandatory that implant supported overdentures has a balanced occlusion witch is meant to protect the implant from the unbalanced impact of premature contacts and occlusal interferences in both static and dynamic positions of the jaw.

The research methods that we have chosen: clinical observation method and experimental method in comparison with two groups:one of study and the other of witness, regarding:

- age of the pacient;

- type of edentation in the lateral area;

- the periode of time in witch the pacient had the lateral edentation;

- type of implant supported overdenture.

The present research is not an institute research but a dental office research, which led to the realistic selection, deliberately targeted of following specific methods of research:

Sample survey -in our research we used a type of questionnaire that we designed, after the Kois's questionnaire model. 
Written questionnaire- is a method of indirect survey research, form of evidence consisting in a battery of questions, called items, written on a special form called questionnaire in connection with a researched topic. The items must have two main qualities: loyalty (constant), which means that they measure the same elements, repeated whenever, and validity, witch means that they maintain the same value of the tested element when is repeated in similar conditions [8-10].

We used questions that may give indications of the existence of certain pathological disorders, developmental disorders that can endanger the health of the TJM.

The mesurement of the articular angle - we used the orthopantomography.

At the top of the articular condyle is drawn a horizontal line parallel to the edge of the negatoscope screen-considered master plan. In parallel is marked a line with a ruler above the anterior slope of the articular condyle.

The angle of intersection between the horizontal and oblique line represents the sagittal condilian guide. According to the literature, this angle varies between 40-60 grd. [4, 9-15]

The bibliographic study - is an indirect method to study the works of the following areas: orthodontics, implant dentistry, radiology, occlusology, statistics, public health,

\section{Results and discussion}

The present survey started from a series of observations taken from pacients with complex clinical cases solved in dental clinics, with implant supported overdentures.

Initial observations of a general nature have been subsequently made a systematic investigation over a period of four years on a small group of patients.

A first conclusion shows that in unilateral ore bilateral edentation we can register specific pathological biomecanical changes of the temporomandibular joint, but also changes regarding the muscle tone, the periarticular components: condyle and meniscus, resulting changes of internal articular anatomy.

This type of implant restoration produces changes in joint biodinamics witch needs to be monitorised quantitatively and qualitatively and compared with physiological joint biomechanics.

The present research is an applied research which is designed to solve short term problems, current issues, and to contribute to the development of an interdisciplinary scientific field represented by the correlation between the proper placed implants (implantology), implant supported overdenture (prosthetics), a functional mastication with healthy temporomandibular joints (functional analysis).

All applied research has been conducted on a sample of 304 subjects aged 18 and over 60 years, in this case the control group has a total of 136 edentulous subjects on one side, but without the presence of any dental implant in the mouth.

The study includes a total of 138 subjects aged 18 and over 60 years, presenting lateral edentulous that have been applied to dental implants and made overdenture. All patients are clinically healthy.

We conclude that the writen questionnaires reveald the following issues: changes in the biomechanics of the temporomandibular joint can be found at very young ages (20-25 years) and are directly linked to the edentation, especially its persistence for a long time, which distort the internal anatomy of the joint.

Patients complain of temporarly or permanently illness signs and joint noise when opening mouth or during chewing. The alarm signal is the occurrence of pain in the joints, which sends patients to the dental office.

Another important point of view is the inadequate or unfinished prosthetic restoration by occlusal equilibration. This problem is quite frequently foud and falls in the etiology of temporomandibular joint biodinamical changes. 
Regarding the orthopantomography analysis method, it reveals that $56 \%$ of the pacients had a previous displacement of the condyle in the glenoid cavity, which leads to a condilien slope angle decrease.

In this survey we study only the propulsion and retro-pulsion motions until the centric relation. The research of the right and left lateral movements will be the main theme of a future study.

Regarding the study group which amounts a total of 138 patients aged 18 and over 60 years, the survey questionnaire method revealed the following problems solved through implant overdenture: implant supported overdenture is an effective method of solving long term edentation.

Gyded Implantology provides optimal placed implants so that the overstrucures can achieve optimal occlusal Equilibration. This helps the joints biomechanics maintaining the health of the temporomandibular joint structures.

Similar with the care of natural teeth supported overdenture, in implant supported overdentures it is mandatory to complete the prosthetic work with correct occlusal equilibration.

Because there is one major difference between the way dental implant acts in the bone structures, compared with natural teeth (dental implant has no mobility and proprioception), it is indicated that from the early prosthetic design plan to be given large importance to joint biomechanics.

Regarding the analysis method orthopantomography reveals that $67 \%$ of the pacients after they recieved the implant supported overdentures, registered a return of the condyle in the glenoidal cavity and the joint symptoms (regarding the pain, deviation and discomfort) disappeared.

This evidence confirms our study, namely that the temporo-mandibular joint toothless occur changes after installing the physiological determined by joint and muscle tone and by biodynamics joint constituents, respectively condyle and meniscus. Toothless implant restoration produces a change in joint biodinamics which must be monitored quantitatively and qualitatively and compared with physiological joint biomechanics and with joint biomechanics of the denture without implant support (Figures 3 and 4).

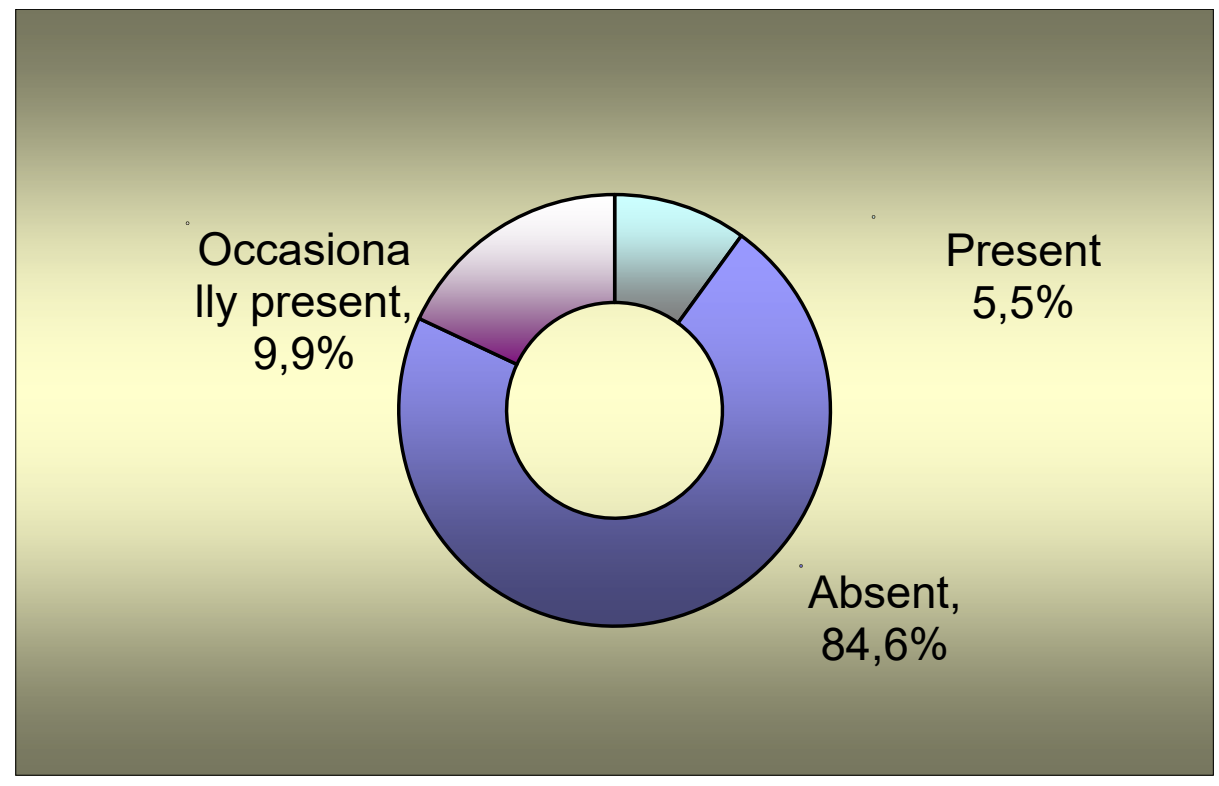

Fig. 3. TJM pain during mouth opening after the proshtetic treatment. 


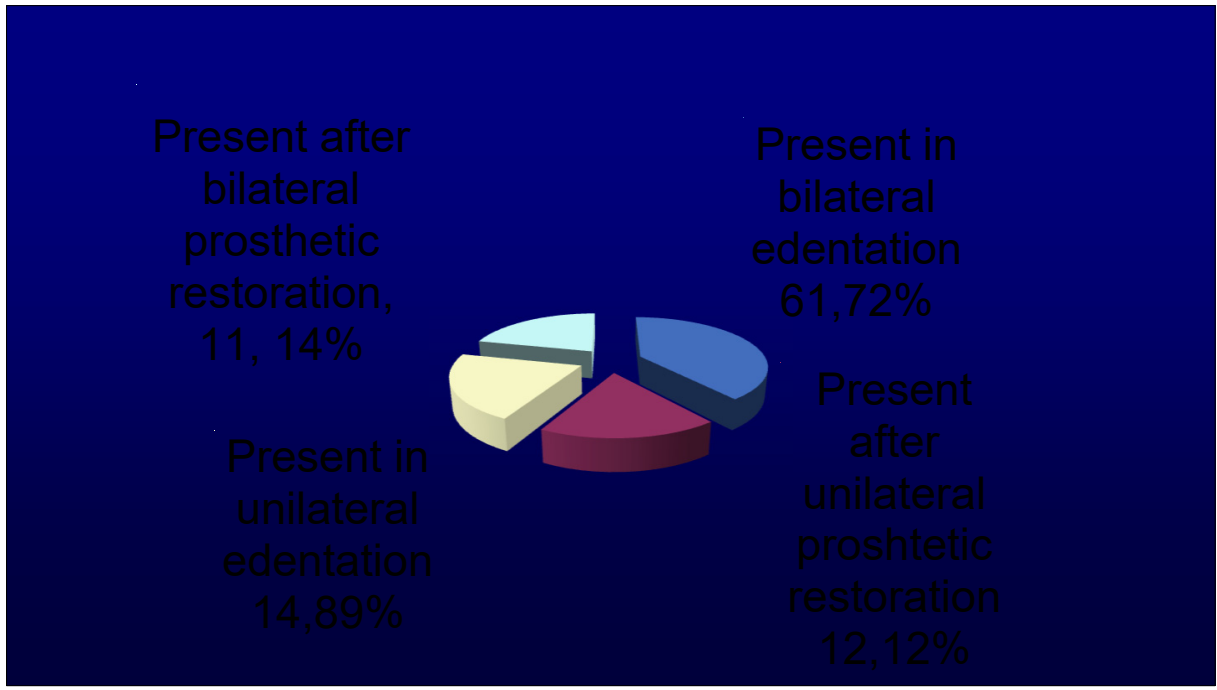

Fig. 4. TJM Crackles during mastication after the proshtetic treatment.

\section{Conclusions and recommendations}

As we noted in our study, statistical results show important changes in the percentage of temporomandibular joint disorder. This reason leads us to the classification of symptoms in "cranio-mandibular disfunction".

In the context of modern dentistry in 2006, our country's integration into European standards is tested according to the directives 78/687/EEC. There are specifically skills for the dentists approved by the European Union countries provided in section 1.5, including: "diagnosis and registration of developmental abnormalities and diseases of the maxillo-facial territory, using existing international classifications".

The profile of the dentist in the European countries has been established by III/D/617/5/86 document.

A patient who recieved implant therapy must be treated as a pacient with a parodontal disease and for this it is required to consider all the consequences regarding the treatment and the position of the implants.

A dental implant involves and requires different biological conditions and thus requires a different behavior compared with that of natural tooth-supported dentures.

It also requires a different medical attitude, in terms of recall observation of the patient that recieved implant supported overdentures.

Another goal is the bacteria plaque control, a matter of great relevance for the sustainability of the dental implant and also for overdenture. It is also required to observ the presence of a possible early inflammatory processes.

If there is an inflammatory process, the therapeutical attitude is very strict to follow:

- $\quad$ It is advisable to periodically perform over-gums scaling only by the hygienist;

- $\quad$ Is totally not suitable for under-gums scaling;

- Indicates the monitoring and observation of any unscrewing or cases without cement;

- $\quad$ The patient is called to regular checks every 3-4 months;

- $\quad$ The patient is investigated radiologically every 12-18 months;

- If an overimplantation inflammatory process occurs, degranulation surgery is reccomended, also detoxification and the possible augmentation of bone defect; 
- $\quad$ Restoration of a 'fixed' implant will take place over 10 to 12 weeks after surgery;

- It is recommended that interventions should be accompanied by the development of CBCT and photos; controls.

The patient is asked to sign a written undertaking engagement about the regular

\section{Refernces}

1. A. Jakstat, Kl. Fktanalyse (2007)

2. A. Garg, Knochen,Biologie, Gewinnung, Transplantation in der zahnarztlichen Implantologie, (2008)

3. F. Bo Rangert, Risikofakt. in der Implantologie (2005)

4. L. Seres Sturm, Anat. stomatologică şi cervico-facială, (1997)

5. I. Cioranu, Chirurgia ATM, (2002)

6. R., Warwick, P., Williams, Gray s anatomy 35th edition,(1973)

7. R., McMinn, Head and neck anatomy,(1981)

8. K., Nieke, Einfurung in die Kieferortopadie, (1995)

9. G., Schmuth, Kieferorthopedie, (1994)

10. S.-H. Lee and D. Terzopoulos, Heads up.biomechanical modeling and neuromuscular control ofthe neck. In Proc. of the ACM SIGGRAPH Conf., pages 1188 - 1198, (2006)

11. A. Maciel, L. Nedel, and C. Freitas, Anatomy basedjoint models for virtual humans skeletons. In Proceedings of Computer Animation, pages 110-116, Geneva, Switzerland, (2002).

12. D. Mattes, D. R. Haynor, H. Vesselle, T. K. Lewellen, and W. Eubank, IEEE Transactions OnMedical Imaging, 22(1):120128, (2003)

13. W. Maurel and D. Thalmann, Computer and Graphics, 24(2):21-24, (2000)

14. G. Monheit and N.A. Badler, IEEE Computer Graphics andApplications, 11(2):29-38, (1991)

15. U. Posselt, Scandinavica, 10(10):19-160, (1952).

16. W. Shao and V. Ng-Thow-Hing, A general jointcomponent framework for realistic articulation in human characters. In Symposium on Interactive 3DGraphics, pages 28-30, (2003)

17. M.B. Villamil, L.P. Nedel, C. Freitas, and A. Maciel, A model to simulate the mastication motion at the temporomandibular joint. In SPIE MEDICAL IMAGING 2005 - Physiology, Function, and Structure from Medical Images, volume 6, San Diego, USA, (2005) 